\title{
Clinical Effectiveness of Percutaneous Epidural Neuro- plasty According to the Type of Single-Level Lumbar Disc Herniation : A 12-Month Follow-Up Study
}

\author{
Pyung Goo Cho, Gyu Yeul Ji, ${ }^{2}$ Young Sul Yoon, ${ }^{3}$ Dong Ah Shin ${ }^{3}$ \\ Department of Neurosurgery, Ajou University College of Medicine, Suwon, Korea \\ Department of Neurosurgery, ${ }^{2}$ Cham Teun Teun Research Institute, Seoul, Korea \\ Department of Neurosurgery, ${ }^{3}$ Yonsei University College of Medicine, Seoul, Korea
}

Objective : To determine whether the outcomes of percutaneous epidural neuroplasty (PEN) are influenced by the type of lumbar disc herniation $(L D H)$ and evaluate the effectiveness of PEN in patients with single-level LDH.

Methods : This study included 430 consecutive patients with single-level LDH who underwent PEN. Before treatment, the LDH type was categorized as bulging, protrusion, extrusion, and sequestration, while Pfirrmann grades were assigned according to imaging findings. Visual analog scale (VAS) scores for back and leg pain and success rates (Odom's criteria) were assessed at 1, 3, 6, and 12 months after treatment.

Results : The mean preoperative VAS scores for back and leg pain were 6.90 and 4.23, respectively; these decreased after PEN as follows : 2.25 and 1.45, respectively, at 1 month; 2.61 and 1.68, respectively, at 3 months; 2.28 and 1.48, respectively, at 6 months; and 2.88 and 1.48 , respectively, at 12 months $(p<0.001)$. The decrease in VAS scores for leg pain was significantly greater in the extrusion and sequestration groups than in the other two groups $(p<0.05)$; there were no other significant differences among groups. More than $70 \%$ patients exhibited good or excellent 12 -month outcomes according to Odom's criteria. Subsequent surgery was required for 59 patients (13.7\%), with a significantly higher rate in the extrusion $(25.0 \%)$ and sequestration (30.0\%) groups than in the bulging $(7.3 \%)$ and protrusion (13.8\%) groups $(p<0.05)$. Nevertheless, subsequent surgery was not required for $>70 \%$ patients with extrusion or sequestration. Patients with Pfirrmann grades 1-3 (14.0-21.5\%) showed a significantly higher rate of subsequent surgery than those with Pfirrmann grade $0(4.9 \% ; p<0.05)$.

Conclusion : Our findings suggest that PEN is an effective treatment for back and leg pain caused by single-level LDH, with the outcomes remaining unaffected by the LDH type.

Key Words : Low back pain · Herniated disc · Percutaneous epidural neuroplasty · Percutaneous adhesiolysis · Treatment outcome.

- Received : March 15, 2019 •Revised : May 6, 2019 •Accepted : May 18, 2019

- Address for reprints : Dong Ah Shin

Department of Neurosurgery, Yonsei University College of Medicine, 50 Yonsei-ro, Seodaemun-gu, Seoul 03722, Korea

Tel : +82-2-2228-2150, Fax : +82-2-393-9979, E-mail : shindongah@me.com, ORCID : https://orcid.org/0000-0002-5225-4083

This is an Open Access article distributed under the terms of the Creative Commons Attribution Non-Commercial License (http://creativecommons.org/licenses/by-nc/4.0) which permits unrestricted non-commercial use, distribution, and reproduction in any medium, provided the original work is properly cited. 


\section{INTRODUCTION}

Back pain affects millions of individuals and is one of the most common conditions prompting patients to seek medical attention ${ }^{2,28}$. Up to $80 \%$ individuals are affected by back pain during their lives, with the prevalence of low back pain increasing by $2-5 \%$ every year ${ }^{1)}$. Furthermore, over $80 \%$ affected patients report recurrent episodes ${ }^{6}$. The National Center for Health Statistics has documented back and spine impairments as the most frequent causes of limitations in daily activities among individuals aged $<45$ years ${ }^{11}$.

Lumbar disc herniation (LDH) occurs in several cases of lumbar nerve root compression, and it accounts for $4 \%$ cases of low back pain. Spinal canal stenosis and post-laminectomy syndrome are also known to compress the lumbar spinal root. In addition, scar tissue formed around nerve roots may irritate them and cause continuous neuropathic pain, while adhesions formed after spine surgery can cause chronic inflammation and nerve root irritation. For patients with low back pain in the absence of neurological abnormalities, conservative treatment with different epidural injection techniques could be useful.

Percutaneous epidural neuroplasty (PEN) is a minimally invasive therapy wherein a catheter is directly placed into the herniated disc or scar tissue compromising the nerve root. In the cervical study, it has been found that PEN treatment improves the patient's visual analog scale (VAS) score and Odom's score $^{23)}$. It has been used for patients with refractory chronic low back pain or failed back surgery syndrome $e^{5,7,8,11,14-17,19,21,27)}$. A systematic review found level I evidence for the effectiveness of percutaneous adhesiolysis in the treatment of chronic refractory low back and lower extremity pain. Accordingly, the authors recommended PEN as a first-line treatment for these conditions ${ }^{14)}$. The goal of PEN is to ameliorate aberrant adhesions, which can physically impede the direct application of drugs around the nerves, and deliver medication to the targeted site ${ }^{4,13,22,29)}$. Thus, this procedure can result in pain reduction and functional improvement in patients with chronic lower back or leg pain due to $\mathrm{LDH}^{20)}$.

However, to the best of our knowledge, no long-term clinical study has observed the outcomes of PEN in patients with $\mathrm{LDH}$ or determined whether the type of LDH influences the outcomes of PEN. Therefore, we conducted the present 12-month follow-up study to determine whether the $\mathrm{LDH}$ type influences the clinical outcomes of PEN and to evaluate the effectiveness of PEN in patients with single-level LDH.

\section{MATERIALS AND METHODS}

The Institutional Review Board of Cham Teun Teun Hospital approved the study (IRB-No. GTIRB-13-005). This retrospective observational study included patients with singlelevel LDH who underwent PEN between February 2010 and March 2011 and were followed-up for $>12$ months at a single hospital. Data were collected from medical charts, radiological images, and surgeons' personal records. The inclusion criteria for the study were as follows : age $>18$ years, diagnosis of single-level LDH with radicular pain or radiculopathic symptoms ineffective to conservative treatment for at least 1 month, and ability to provide written informed consent for participation in a clinical trial when the presence of adhesion was suspected. Patients with multi-level LDH, lumbar stenosis, spinal cord lesions, hyaluronic acid-sensitive side effects, uncontrolled diabetes, and previous lumbar surgeries were excluded.

Single-level LDH was diagnosed on the basis of clinical symptoms, neurological examinations, and imaging studies including radiography and magnetic resonance imaging (MRI). MRI findings were analyzed for the type of LDH and Pfirrmann grade ${ }^{25}$.

The LDH type was categorized as follows : bulging, defined as "an out-pouching not compressed against the nerves and occurring with the spinal disc and associated ligaments intact" ( $n=124)$; protrusion, defined as "an out-pouching pressed against the nerves and occurring with the spinal disc and associated ligaments intact" ( $\mathrm{n}=240$ ); extrusion, defined as "disc extrusion where the outer part of the spinal disc is ruptured, allowing the inner, gelatinous part of the disc to squeeze out" $(\mathrm{n}=56)$; and sequestration, defined as "disc sequestration where the center, gelatinous portion of the disc is not only squeezed out but also separated from the main part of the disc" ( $\mathrm{n}=10)$. The Pfirrmann grade was assigned as follows: grade 0 , normal, "no compromise of the nerve root is seen" $(\mathrm{n}=123)$; grade 1 , contact, "visible contact of disc material with the nerve root without evidence of the normal epidural fat layer between the two" ( $\mathrm{n}=86)$; grade 2, deviation, "nerve root displaced dorsally by disc material" $(\mathrm{n}=128)$; and grade 3 , compression, "compressed nerve root between the disc mate- 
rial and the wall of the spinal canal; it may appear flattened or may be indistinguishable from the disc material" $(\mathrm{n}=93)$.

The VAS (score range, 0 to 10 , with 0 reflecting no pain) for back pain (VAS back) and leg pain (VAS leg), as well as the clinical outcome based on Odom's criteria, which classifies outcomes as excellent, good, fair, or poor, were used to evaluate the clinical effectiveness of PEN with regard to pain reduction and functional improvement. VAS scores were recorded before and 1, 3, 6, and 12 months after PEN, while clinical outcomes were evaluated 1, 3, 6, and 12 months after PEN. All patients were asked to provide responses based on the average severity of symptoms over the week prior to their visit. Successful pain relief was described as a $\geq 50 \%$ decrease in the VAS score, while good or excellent outcomes according to Odom's criteria were considered to reflect a "good" outcome.

PEN was performed under fluoroscopic guidance in a sterile operating room with monitoring equipment for blood pressure, pulse rate, and pulse oximetry. The fluoroscope was adjusted over the lumbosacral area such that a caudal approach could be used in both the anteroposterior and lateral views. Following appropriate positioning of the fluoroscope, the needle insertion site was determined around the sacral hiatus and was injected with local anesthetics. An $\mathrm{RK}^{\circledR}$ needle
(Epimed International Inc., Gloversville, NY, USA) was introduced into the caudal epidural space under fluoroscopic guidance. On confirmation of the needle position in the epidural space, lumbar epidurography was performed with approximately $5 \mathrm{~mL}$ of a noniodinated contrast agent (IOBRIX ${ }^{\circledR}, \mathrm{AC}-$ CUZEN, Seoul, Korea). Filling defects were identified through observation of contrast flow into the nerve root (Fig. 1). We confirmed that there was no intravascular or subarachnoid placement of the needle; if such malpositioning was observed, the needle was repositioned. Following epidurography and confirmation of appropriate needle positioning, a Racz catheter (Epimed International Inc.) was advanced through the RK needle to the area of the filling defect or the site of pathology as determined by MRI. Then, adhesiolysis was performed, after which final positioning of the catheter was achieved in the lateral and ventral epidural spaces. Following satisfactory positioning of the catheter, at least $3 \mathrm{~mL}$ of contrast agent was injected. If there was no subarachnoid, intravascular, or other extra-epidural filling, and if satisfactory filling was observed in the target regions, $6 \mathrm{~mL}$ of $0.2 \%$ preservative-free ropivacaine containing 1500 units of hyaluronidase and $4 \mathrm{~mL}$ of betamethasone sodium phosphate was injected. An hour after the procedure, $6 \mathrm{~mL}$ of $8 \%$ sodium chloride solution was in-

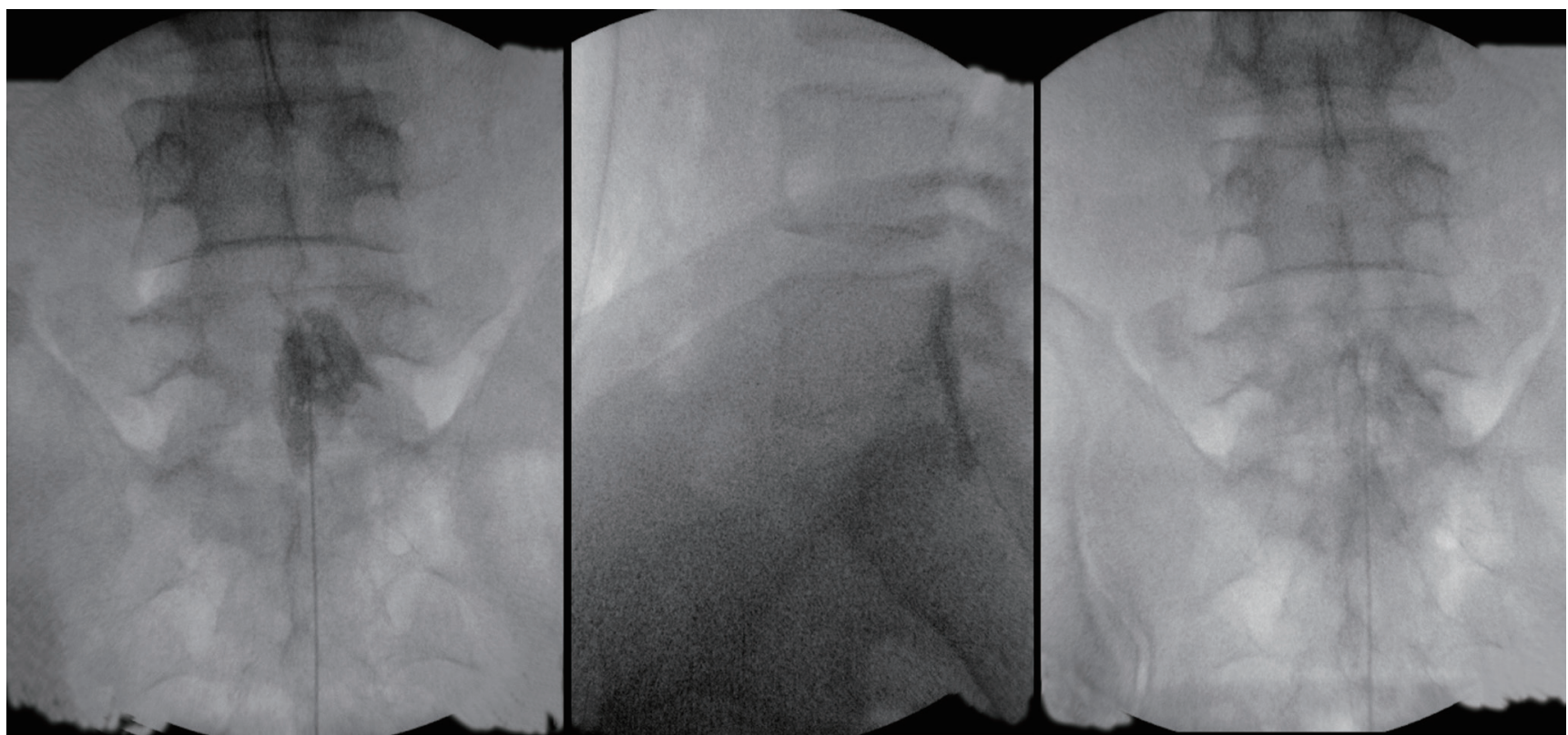

Fig. 1. A specially designed spring-wire catheter (Racz catheter ${ }^{\oplus}$; Epimed International Inc., Gloversville, NY, USA) needle was introduced into the sacral epidural space under fluoroscopy. Once the needle placement was confirmed to be in the epidural space, a lumbar epidurogram was carried out with contrast. Identification of the filling defects was carried out by examining the contrast flow into the nerve roots. After appropriate determination of epidurography, a Racz catheter was slowly passed through the needle to the intended area, and adhesiolysis and scheduled injection were carried out. 
fused over 30 minutes under monitoring in the recovery room. The intravenous line and epidural catheter were removed, and the patient was discharged when all parameters were observed to be satisfactory. The first follow-up visit was scheduled at 2 weeks after the procedure. During these 2

Table 1. Demographic data

\begin{tabular}{lc}
\hline Case & Value $(\mathbf{n}=\mathbf{4 3 0})$ \\
\hline Male ratio & $213(49.5)$ \\
Mean age & $46.7 \pm 13.8$ \\
Symptom duration (months) & $2.8 \pm 0.5$ \\
Level & \\
L1/2 & $4(0.93)$ \\
L2/3 & $4(0.93)$ \\
L3/4 & $30(6.98)$ \\
L4/5 & $269(62.56)$ \\
L5/S1 & $123(28.60)$ \\
Comorbidity & \\
Hypertension & $77(17.91)$ \\
Diabetes mellitus & $126(29.03)$ \\
Tuberculosis & $11(2.56)$ \\
Hepatitis & $13(3.02)$ \\
Complete follow-up patients & \\
1 month & 430 \\
3 months & 408 \\
6 months & 385 \\
12 months & 371 \\
\hline
\end{tabular}

Values are presented as mean \pm standard deviation or number (\%) weeks, all patients received nonsteroidal anti-inflammatory drugs and muscle relaxants in equal doses to reduce procedure-related pain.

Student's t-test, analysis of variance (ANOVA), and the chisquare test were used to estimate the reliability of the radiological and clinical outcomes. Student's t-test was used to compare demographic data between pairs of groups; ANOVA was used to compare VAS back and leg scores, Pfirrmann grades, success rates (Odom's criteria), and symptom duration among the LDH types; and the chi-square test was used to compare VAS back and leg scores and the rates of hypertension, diabetes mellitus, tuberculosis, and hepatitis among the LDH types. All statistical analyses were performed using SPSS software (SPSS Inc., Chicago, IL, USA). A $p$-value of $<0.05$ was considered statistically significant.

\section{RESULTS}

In total, 430 patients were enrolled. One-, 3-, 6-, and 12-month follow-up data were available for 430, 408, 385, and 371 patients, respectively. Table 1 summarizes the demographic data for these patients. The average age at treatment was 46.7 years, and the men versus women ratio was $49.5 \%$. Consecutive target levels were as follows : $\mathrm{L} 1 / 2$ and $\mathrm{L} 2 / 3, \mathrm{n}=4 ; \mathrm{L} 3 / 4$, $\mathrm{n}=30 ; \mathrm{L} 4 / 5, \mathrm{n}=269$; and L5/S1, $\mathrm{n}=123$. Among these patients, $375(87.2 \%)$ received a single nerve root block prior to adhesiolysis.

With regard to the LDH type, there were 124 patients with

Table 2. Demographic data according to the type of single level disc herniation

\begin{tabular}{|c|c|c|c|c|c|}
\hline Category & Bulging & Protrusion & Extrusion & Sequestration & Total \\
\hline Cases & 124 & 240 & 56 & 10 & 430 \\
\hline Male ratio & $56(45.16)$ & 115 (47.92) & $36(64.29)$ & $6(60.00)$ & $213(49.50)$ \\
\hline Mean age & $51.5 \pm 13.3$ & $45.4 \pm 13.6$ & $46.7 \pm 13.8$ & $46.7 \pm 13.8$ & $46.7 \pm 13.8$ \\
\hline Symptom duration (months) & $3.2 \pm 0.2$ & $2.7 \pm 0.5$ & $3.1 \pm 0.4$ & $1.9 \pm 0.1$ & $2.8 \pm 0.5$ \\
\hline \multicolumn{6}{|l|}{ Complete follow-up } \\
\hline 1 month & 124 & 240 & 56 & 10 & 430 \\
\hline 3 months & 120 & 232 & 47 & 9 & 408 \\
\hline 6 months & 117 & 215 & 45 & 8 & 385 \\
\hline 12 months & 115 & 207 & 42 & 7 & 371 \\
\hline Operated cases & $9(7.26)$ & $33(13.75)$ & $14(25.00)$ & $3(30.00)$ & 59 (13.72) \\
\hline
\end{tabular}

Values are presented as mean \pm standard deviation or number (\%) 
bulging, 240 with protrusion, 56 with extrusion type, and 10 with sequestration (Table 2). The men versus women ratios and mean age were $45.2 \%$ and $51.5 \pm 13.3$ years, respectively, in the bulging group; $47.9 \%$ and $45.4 \pm 13.6$ years, respectively, in the protrusion group; $64.3 \%$ and $46.7 \pm 13.8$ years, respectively, in the extrusion group; and $60.0 \%$ and $46.7 \pm 13.8$ years, respectively, in the sequestration group. The men versus women ratio was significantly different between the bulging and extrusion groups and the protrusion and extrusion groups, while the mean age was significantly different between the bulging and protrusion groups and the bulging and extrusion groups. There were no other significant differences. A total of 59 patients (13.7\%) underwent subsequent surgical decompression for severe pain during the follow-up period, and their clinical data were excluded from the date of the surgery. Nine (7.3\%), 33 (13.8\%), 14 (25.0\%), and three (30.0\%) patients with bulging protrusion, extrusion, and sequestration, respectively, required surgical decompression during the follow-up period.
Thus, 12-month clinical follow-up data were available for 115 (92.7\%), 207 (86.3\%), 42 (75.0\%), and seven (70.0\%) patients with bulging, protrusion, extrusion, and sequestration, respectively (Fig. 2). The rate of subsequent surgery was relatively high for the extrusion and sequestration groups, with significant differences between the bulging and extrusion groups ( $p<0.001)$, bulging and sequestration groups $(p=0.015)$, and protrusion and extrusion groups $(p=0.038)$. Nevertheless, $>70 \%$ patients in the extrusion and sequestration groups did not require surgery during the 12-month follow-up period.

With regard to the Pfirrmann grade, there were 123 patients with Pfirrmann grade 0, 86 with Pfirrmann grade 1, 128 with Pfirrmann grade 2, and 93 with Pfirrmann grade 3. Surgical decompression during the 12-month follow-up period was required for six (4.88\%), 12 (13.95\%), 21 (16.41\%), and 20 (21.51\%) patients with Pfirrmann grades $0,1,2$, and 3, respectively. Thus, the rate of subsequent surgery according to the Pfirrmann grade was significantly different between patients

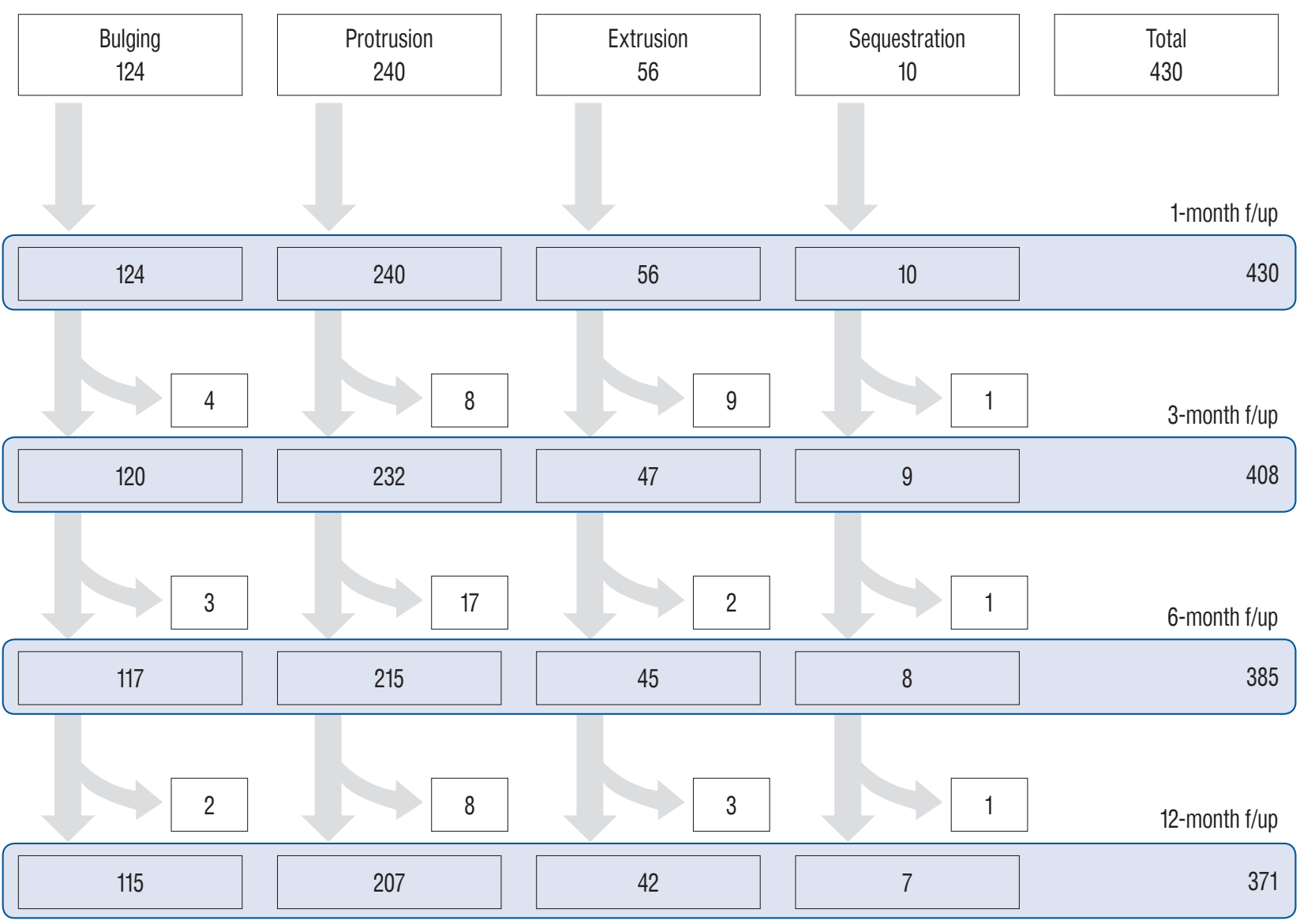

Fig. 2. Flow sheet of surgical decompression and follow-up during 12 months. f/up : follow-up. 
with grade 0 and those with grades $1(p=0.021), 2(p=0.003)$, and $3(p<0.001)$.

The results of clinical assessments are summarized in Figs. 3-6. The overall mean VAS scores for back and leg pain were 6.9 and 4.2 , respectively, before treatment; 2.3 and 1.5 , respectively, at 1 month; 2.6 and 1.7, respectively, at 3 months; 2.3 and 1.5 , respectively, at 6 months; and 2.9 and 1.5 , respectively, at 12 months (all $p<0.001$ vs. the preoperative scores, Fig. 3). With regard to the LDH type, the mean preoperative VAS scores for back and leg pain improved at 12 months after treatment as follows (Fig. 4) : 6.9 and 3.9 to 2.9 and 1.5, respec-

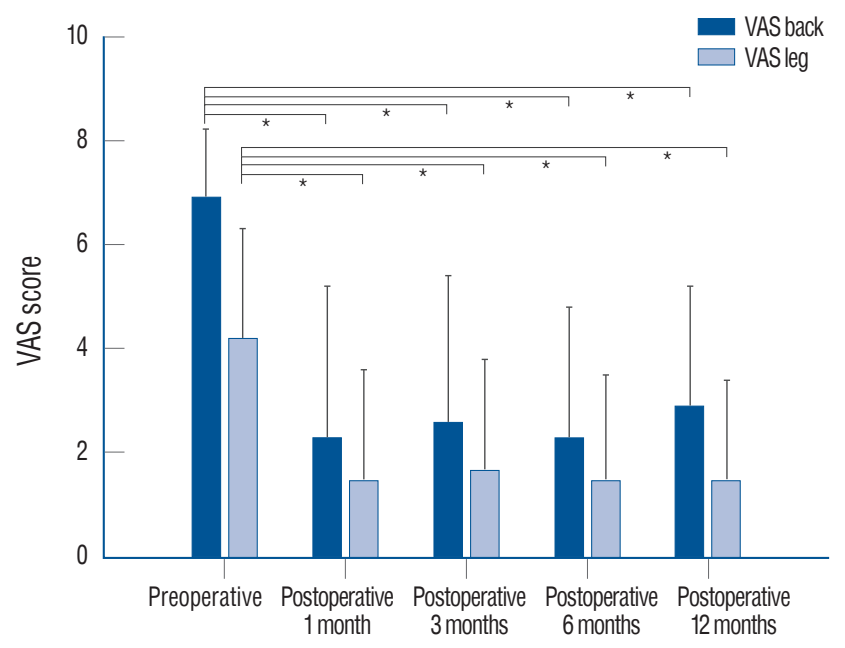

Fig. 3. Total mean VAS scores for back and leg pain were significantly less than those preoperatively after percutaneous epidural neuroplasty. *Indicates a statistical difference of less than 0.050 . VAS : visual analog scale.

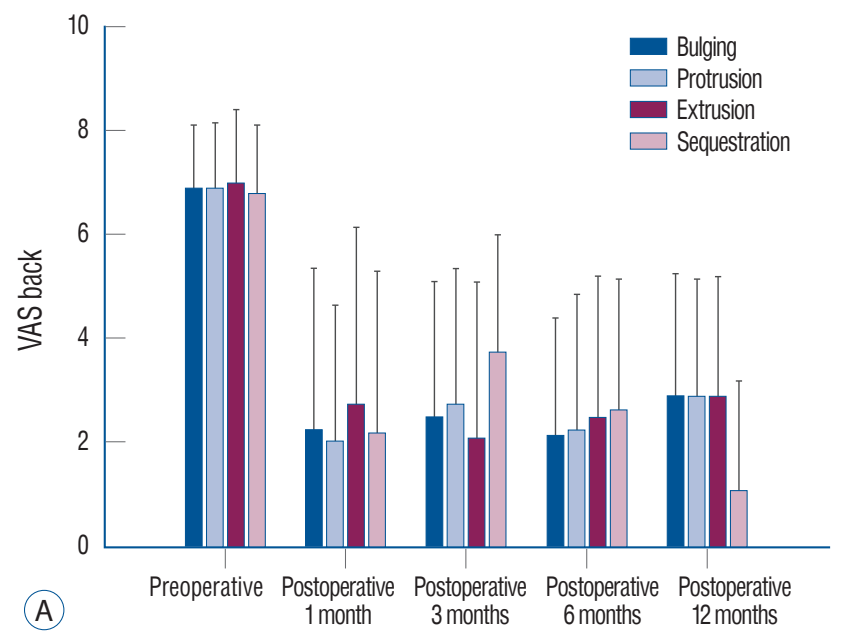

tively, in the bulging group; 6.9 and 4.3 to 2.9 and 1.4 , respectively, in the protrusion group; 7.0 and 4.8 to 2.9 and 2.0 , respectively, in the extrusion group; and 6.8 and 4.0 to 1.1 and 0.0 , respectively, in the sequestration group (all $p<0.001 \mathrm{vs}$. the preoperative scores). With regard to the Pfirrmann grade, the mean preoperative VAS scores for back and leg pain improved at 12 months after treatment as follows (Fig. 5) : 6.9 and 3.5 to 2.9 and 1.3 , respectively, in the grade 0 group; 6.9 and 4.1 to 2.4 and 1.2, respectively, in the grade 1 group; 6.9 and 4.6 to 3.0 and 1.6, respectively, in the grade 2 group; and 6.8 and 4.9 to 2.4 and 1.9, respectively, in the grade 3 group (all $p<0.001$ vs. the preoperative score). The overall rates of a "good" outcome, defined by Odom's criteria for a good or excellent outcome, were $81.3 \%$ at 1 month, $73.1 \%$ at 3 months, $74.8 \%$ at 6 months, and $71.7 \%$ at 12 months after PEN (Fig. 6), with no significant differences among the LDH types $(p=0.104)$.

\section{DISCUSSION}

In the present study, we found that PEN is an effective treatment for back and leg pain caused by single-level LDH, regardless of the LDH type.

PEN has been used to treat chronic back pain refractory to other conservative management strategies and has been shown to have good clinical efficacy ${ }^{31)}$. In fact, the procedure has demonstrated superior effectiveness over not only physical therapy $^{30)}$ but also caudal epidural steroid injections for the

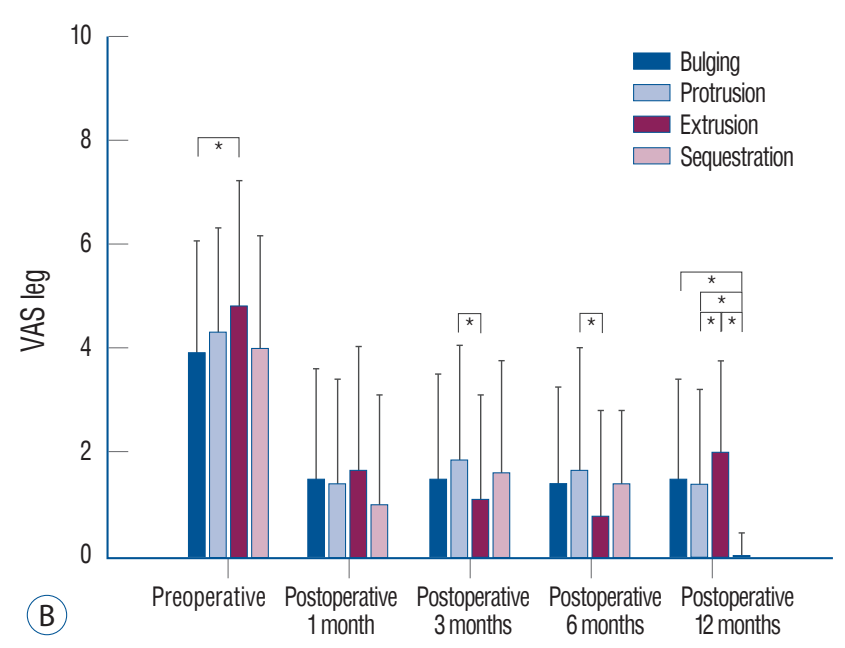

Fig. 4. Mean VAS scores for back pain (A) and leg pain (B) according to lumbar disc herniation type during 12 months of follow-up. *Indicates a statistical difference of less than 0.050 between groups. VAS : visual analog scale. 
treatment of chronic lower back and leg pain ${ }^{16,21)}$. This can be attributed to the ability of PEN to eliminate adhesions or fibrous tissue that can impede the spread of medications injected into the specific lesion site. Moreover, PEN allows for the placement of the catheter tip within the target area. These factors enable the delivery of an adequate concentration of steroid or other solution to the appropriate target area ${ }^{5)}$. However, PEN is considered more invasive and expensive than are conventional blocks; therefore, it is usually performed for patients who fail to respond to conservative treatment and conventional injections. Although a previous study by Lee and Lee $^{12)}$ has attempted to evaluate the efficacy of PEN in patients unresponsive to transforaminal epidural injections, to the best of our knowledge, no long-term studies have attempted to assess the clinical outcomes of PEN according to the type of LDH.

In the present study, patients were categorized according to the LDH type (bulging, protrusion, extrusion, and sequestration) and Pfirrmann grade (grades $0-3$ ). The results revealed similar clinical outcomes for all patients, regardless of the LDH type or Pfirrmann grade. The only difference was observed in the rate of subsequent surgical decompression during the 12-month follow-up period; patients with extrusion or sequestration and those with a high Pfirrmann grade exhibited higher rates. These results are similar to those of Lee and Lee $^{12)}$, who categorized LDH as follows : bulging, protrusion, and extrusion; central, subarticular, and foraminal location; and abutment, displacement, and compression of root. They found that the LDH type did not influence the PEN outcome at 2 weeks and 3 months. In the present 12-month follow-up study, surgical decompression during the follow-up period was required for $7.3 \%$ patients with bulging, $13.8 \%$ patients with protrusion, $25.0 \%$ patients with extrusion, $30.0 \%$ patients with sequestration, $4.9 \%$ patients with Pfirrmann grade 0, $14.0 \%$ patients with Pfirrmann grade 1, 16.4\% patients with Pfirrmann grade 2, and 21.5\% patients with Pfirrmann grade 3. Although the rate of subsequent surgery differed significantly between the bulging and extrusion groups, bulging and sequestration groups, protrusion and extrusion groups, and

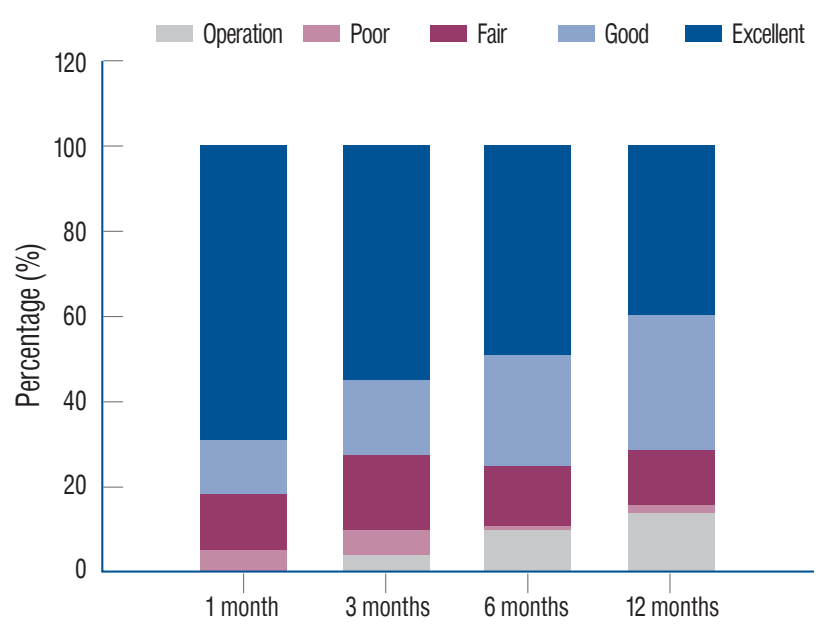

Fig. 6. Overall success rates fulfilling Odom's criteria of "good" and "excellent" outcomes were $81.3 \%$ at 1 month, $73.1 \%$ at 3 months, $74.8 \%$ at 6 months, and $71.7 \%$ at 12 months after percutaneous epidural neuroplasty treatment.
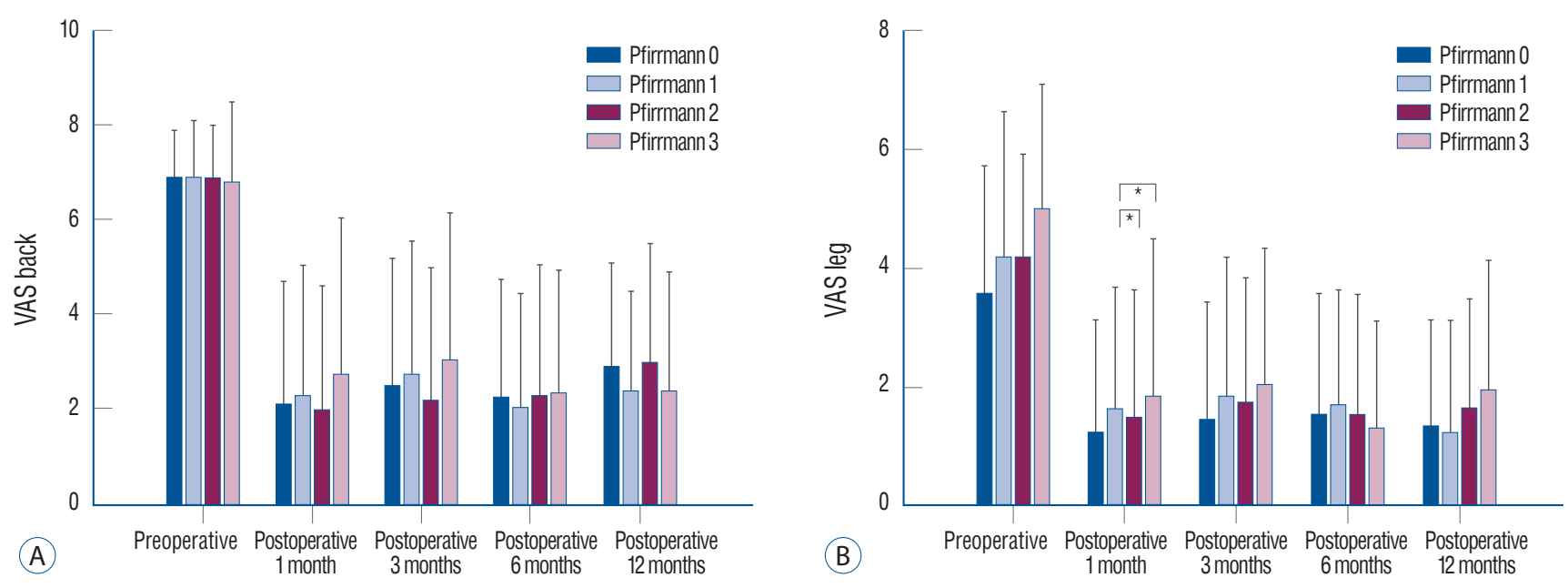

Fig. 5. Mean VAS scores for back pain (A) and leg pain (B) according to Pfirrmann grade during 12 months of follow-up. *Indicates a statistical difference of less than 0.050 between groups. VAS : visual analog scale. 
Pfirrmann grade 0 and other Pfirrmann grade groups, $>70 \%$ patients with extrusion or sequestration did not require surgical treatment during the 12-month follow-up period after PEN. Extrusion and sequestrated type discs may not require surgical treatment because self-resolution occurs more often than other types ${ }^{26}$. In our clinical set-up, we usually recommend surgical treatment to patients with extrusion or sequestration, because we believe that surgery is more effective than PEN in these cases. However, some of these patients refused surgical treatment and were consequently included in our study. Therefore, the number of patients with extrusion or sequestration was smaller in the present study than in other studies $^{3,24)}$, and, consequently, a high rate of subsequent surgery was recorded for these patients. This outcome indicated that surgery is a good treatment modality for such cases; however, a relatively high percentage of patients who are generally recommended surgical treatment demonstrated very impressive results with PEN. This suggests that surgery may not always be necessary for such cases.

In the present study, we investigated the clinical outcomes of PEN not only according to the type of single-level LDH but also the efficacy of PEN. All patients demonstrated significant improvements in VAS scores for back pain and leg pain at 12 months after PEN. The overall success rates based on Odom's criteria for good and excellent outcomes were also $>70 \%$ during the 12-month follow-up period. Studies on the clinical efficacy of PEN have reported that the procedure offers more significant pain reduction and better functional outcomes than do medication, physical therapy, and exercise programs ${ }^{9,18,30)}$. Heavner et al..$^{9)}$ suggested that approximately $49 \%$ of patients who failed to achieve clinical improvement via other conservative treatments showed a significant decrease in their pain scores at 3 months, while $43 \%$ and $49 \%$ reported pain reduction at 6 and 12 months, respectively. A few studies have also compared the efficacy of PEN with that of epidural steroid injections. Specifically, two reports have identified that the clinical outcomes of PEN are better than those of caudal epidural injections in patients with spinal stenosis and lumbar postlaminectomy syndrome ${ }^{16,211}$. This efficacy was also observed in the present study of patients with single-level LDH, who demonstrated significantly improved VAS scores for leg and back pain at 12 months after PEN. The overall success rate also remained $>70 \%$ during the 12-month follow-up period. A previous study by Lee and Lee ${ }^{12)}$ also reported signifi- cant improvements in VAS back, VAS leg, and Oswestry Disability Index scores at 3 months after PEN. However, some studies have shown different results. Recently, Helm Ii et al. ${ }^{10)}$ conducted a systematic review to evaluate the efficacy of PEN for the management of chronic low back pain in patients with post-lumbar surgery syndrome and spinal stenosis. The authors included five randomized controlled trials and two observational studies and found fair evidence of the effectiveness of PEN in providing relief from low back and/or leg pain caused by post-lumbar surgery syndrome or spinal stenosis. However, they also reported a low rate of complications that were generally minimal and self-limiting. Therefore, well-designed randomized controlled studies as well as systematic reviews of such studies are necessary for resolution of controversies concerning the efficacy of PEN.

This study has several limitations. First, it was a retrospective analysis. Second, a control group was not included for comparison, so improved clinical outcomes after PEN could not be distinguished from the natural course of LDH. Third, the distribution of patients according to the level and type of LDH was uneven, and this may have affected the statistical results. Fourth, this study did not take the MRI after procedure for checking the spontaneous regression of disc. This regression can affect the PEN effect according the disc type. Despite these limitations, however, our findings have some important clinical implications. We initially considered that the efficacy of PEN would differ with the type of LDH and Pfirrmann grade, whereas the actual findings contradicted our hypothesis. In fact, the clinical outcomes of extrusion and sequestration, which we generally consider as strong predictors of a poor outcome after PEN, were found to be excellent.

\section{CONCLUSION}

The findings of this study suggest that PEN is an effective treatment strategy for patients with back and leg pain caused by single-level LDH who do not respond to conservative treatment, thus negating the need for lumbar surgery. Moreover, they indicate that the efficacy of PEN remains unaffected by the type of single-level LDH. 


\section{CONFLICTS OF INTEREST}

No potential conflict of interest relevant to this article was reported.

\section{INFORMED CONSENT}

Informed consent was obtained from all individual participants included in this study.

\section{AUTHOR CONTRIBUTIONS}

\author{
Conceptualization : DAS \\ Data curation : GYJ \\ Formal analysis : PGC \\ Funding acquisition : YSY \\ Methodology : PGC \\ Project administration : PGC, DAS \\ Visualization : GYJ \\ Writing - original draft : PGC, DAS \\ Writing - review \& editing : GYJ, YSY, PGC, DAS
}

\section{References}

1. Canale ST BJ : Campbell's operative orthopaedics. Philadelphia : Elsevier, 2003

2. Carey TS, Garrett JM, Jackman AM : Beyond the good prognosis. Examination of an inception cohort of patients with chronic low back pain. Spine (Phila Pa 1976) 25 : 115-120, 2000

3. Carragee EJ, Han MY, Suen PW, Kim D : Clinical outcomes after lumbar discectomy for sciatica: the effects of fragment type and anular competence. J Bone Joint Surg Am 85 : 102-108, 2003

4. Chopra P, Smith HS, Deer TR, Bowman RC : Role of adhesiolysis in the management of chronic spinal pain: a systematic review of effectiveness and complications. Pain Physician 8 : 87-100, 2005

5. Epter RS, Helm S 2nd, Hayek SM, Benyamin RM, Smith HS, Abdi S : Systematic review of percutaneous adhesiolysis and management of chronic low back pain in post lumbar surgery syndrome. Pain Physician 12 361-378, 2009

6. Franklin CVJ, Kalirathinam D, Palekar T, Nathani N : Effectiveness of PNF training for chronic low back pain. IOSR JNHS 2 : 41-52, 2013

7. Han YJ, Lee MN, Cho MJ, Park HJ, Moon DE, Kim YH : Contrast runoff correlates with the clinical outcome of cervical epidural neuroplasty using a racz catheter. Pain Physician 19 : E1035-E1040, 2016
8. Hayek SM, Helm S, Benyamin RM, Singh V, Bryce DA, Smith HS : Effectiveness of spinal endoscopic adhesiolysis in post lumbar surgery syndrome: a systematic review. Pain Physician 12 : 419-435, 2009

9. Heavner JE, Racz GB, Raj P : Percutaneous epidural neuroplasty: prospective evaluation of $0.9 \% \mathrm{NaCl}$ versus $10 \% \mathrm{NaCl}$ with or without hyaluronidase. Reg Anesth Pain Med 24 : 202-207, 1999

10. Helm li S, Benyamin RM, Chopra P, Deer TR, Justiz R : Percutaneous adhesiolysis in the management of chronic low back pain in post lumbar surgery syndrome and spinal stenosis: a systematic review. Pain Physician 15 : E435-E462, 2012

11. Ji GY, Oh CH, Moon B, Choi SH, Shin DA, Yoon YS, et al. : Efficacy of percutaneous epidural neuroplasty does not correlate with dural sac cross-sectional area in single level disc disease. Yonsei Med J 56 : 691-697, 2015

12. Lee JH, Lee SH : Clinical effectiveness of percutaneous adhesiolysis using Navicath for the management of chronic pain due to lumbosacral disc herniation. Pain Physician 15 : 213-221, 2012

13. Manchikanti L, Bakhit CE : Percutaneous lysis of epidural adhesions. Pain Physician 3 : 46-64, 2000

14. Manchikanti L, Boswell MV, Datta S, Fellows B, Abdi S, Singh V, et al. : Comprehensive review of therapeutic interventions in managing chronic spinal pain. Pain Physician 12 : E123-E198, 2009

15. Manchikanti L, Boswell MV, Singh V, Benyamin RM, Fellows B, Abdi $S$, et al. : Comprehensive evidence-based guidelines for interventional techniques in the management of chronic spinal pain. Pain Physician 12 : 699-802, 2009

16. Manchikanti L, Cash KA, McManus CD, Pampati V, Singh V, Benyamin R : The preliminary results of a comparative effectiveness evaluation of adhesiolysis and caudal epidural injections in managing chronic low back pain secondary to spinal stenosis: a randomized, equivalence controlled trial. Pain Physician 12 : E341-E354, 2009

17. Manchikanti L, Manchikanti KN, Gharibo CG, Kaye AD : Efficacy of percutaneous adhesiolysis in the treatment of lumbar post surgery syndrome. Anesth Pain Med 6 : e26172, 2016

18. Manchikanti L, Pampati V, Fellows B, Rivera J, Beyer CD, Damron KS : Role of one day epidural adhesiolysis in management of chronic low back pain: a randomized clinical trial. Pain Physician 4 : 153-166, 2001

19. Manchikanti L, Pampati V, Fellows B, Rivera JJ, Damron KS, Beyer C, et al. : Effectiveness of percutaneous adhesiolysis with hypertonic saline neurolysis in refractory spinal stenosis. Pain Physician 4 : 366-373, 2001

20. Manchikanti L, Singh V, Bakhit CE, Fellows B : Interventional techniques in the management of chronic pain: part 1.0. Pain Physician $3: 7-42$, 2000

21. Manchikanti L, Singh V, Cash KA, Pampati V, Datta S : A comparative effectiveness evaluation of percutaneous adhesiolysis and epidural steroid injections in managing lumbar post surgery syndrome: a randomized, equivalence controlled trial. Pain Physician 12 : E355-E368, 2009

22. Manchikanti L, Staats PS, Singh V, Schultz DM, Vilims BD, Jasper JF, et al. : Evidence-based practice guidelines for interventional techniques in 
the management of chronic spinal pain. Pain Physician $6: 3-81,2003$

23. Oh CH, Ji GY, Cho PG, Choi WS, Shin DA, Kim KN, et al. : The catheter tip position and effects of percutaneous epidural neuroplasty in patients with lumbar disc disease during 6-months of follow-up. Pain Physician 17 : E599-E608, 2014

24. Peul WC, van Houwelingen HC, van den Hout WB, Brand R, Eekhof JA, Tans JT, et al. : Surgery versus prolonged conservative treatment for sciatica. N Engl J Med 356 : 2245-2256, 2007

25. Pfirrmann CW, Dora C, Schmid MR, Zanetti M, Hodler J, Boos N : MR image-based grading of lumbar nerve root compromise due to disk herniation: reliability study with surgical correlation. Radiology 230 : 583588, 2004

26. Splendiani A, Puglielli E, De Amicis R, Barile A, Masciocchi C, Gallucci M : Spontaneous resolution of lumbar disk herniation: predictive signs for prognostic evaluation. Neuroradiology 46 : 916-922, 2004

27. Taheri A, Khajenasiri AR, Nazemian Yazdi NA, Safari S, Sadeghi J, Hata- mi M : Clinical evaluation of percutaneous caudal epidural adhesiolysis with the racz technique for low back pain due to contained disc herniation. Anesth Pain Med 6 : e26749, 2016

28. Trainor TJ, Wiesel SW : Epidemiology of back pain in the athlete. Clin Sports Med 21 : 93-103, 2002

29. Trescot AM, Chopra P, Abdi S, Datta S, Schultz DM : Systematic review of effectiveness and complications of adhesiolysis in the management of chronic spinal pain: an update. Pain Physician 10 : 129-146, 2007

30. Veihelmann A, Devens C, Trouillier H, Birkenmaier C, Gerdesmeyer L, Refior $\mathrm{HJ}$ : Epidural neuroplasty versus physiotherapy to relieve pain in patients with sciatica: a prospective randomized blinded clinical trial. J Orthop Sci $11:$ 365-369, 2006

31. Viesca CO, Racz GB, Day MR : Special techniques in pain management: lysis of adhesions. Anesthesiol Clin North Am 21 : 745-766, vi, 2003 